\title{
Fibre grating refractometer sensors for composite process monitoring
}

\author{
Stephen J Buggy ${ }^{1}$, Edmond Chehura ${ }^{1}$, Alexandros A Skordos ${ }^{2}$, Athanasios Dimopoulos ${ }^{2}$ \\ Stephen W James ${ }^{1}$, Ivana K Partridge ${ }^{2}$, Ralph P Tatam ${ }^{1 *}$ \\ ${ }^{1}$ Engineering Photonics Group, School of Engineering \\ ${ }^{2}$ Composites Centre, School of Applied Sciences \\ Cranfield University, Cranfield, Bedfordshire, England, MK43 0AL
}

\begin{abstract}
Optimum performance from advanced composites requires careful control of the resin matrix during cure. This is to ensure there are no cure induced voids and to minimise the build up of internal stresses. Careful control of the process is also necessary to reduce wastage. Traditional resin inspection techniques are bulk or sample oriented and thus cannot provide data about critical component parts. Optical fibre based sensors however, allow for in-situ monitoring techniques to be deployed in components without effecting their structural integrity.

In this work, two fibre optic grating techniques are demonstrated as process monitoring sensors and are compared with a Fresnel refractometric method. The change in refractive index of a resin has previously been used as a means for assessing the degree of cure. The central wavelength of an attenuation band of a long period grating (LPG) was monitored during the cure of a resin. In parallel the spectral resonances of a tilted fibre Bragg grating (FBG) are also monitored. The two techniques are shown to correlate well with the Fresnel based method in both detecting the resin and monitoring the state of cure, indicating the potential of the techniques for online production monitoring.
\end{abstract}

Keywords: Long period grating, tilted fibre Bragg grating, Fresnel refractometer, resin cure monitoring, differential scanning calorimetry, impedance spectroscopy

\section{INTRODUCTION}

A number of fibre optic methods for on-line monitoring of the cure of epoxy resins have been reported [1]. They offer clear advantages over traditional monitoring techniques, due to their inherently small dimensions and compatibility with fibre reinforced plastics. Typically, the optical fibre is used as the means of delivering the light to and from a sample, e.g. spectroscopic methods [1-3]. Utilising the fibre as an evanescent wave sensing element has also been reported using fluorescence [4-6]. Direct interaction of the propagating mode with the surrounding environment, is often exploited, achieved by etching or side polishing the optical fibre to remove the cladding and thus expose the evanescent tail of the core mode field [7]. Techniques that do not require removal of the fibre cladding and hence retain their mechanical integrity are useful in applications requiring embedding of the optical fibre, such as the monitoring of the cure of composite materials. In-fibre grating structures are capable of coupling light from the core of the fibre into the cladding, where the interaction of the cladding modes with the external environment enables the measurement of the surrounding refractive index without the need for etching or side polishing of the fibre. Two in-fibre grating structures which act as core to cladding mode couplers are considered here, the long period grating (LPG) and the tilted fibre Bragg grating (TFBG). An LPG produces a discrete set of attenuation bands in the transmission spectrum of the optical fibre [8]. The refractive index sensitivity of LPGs has been exploited for a wide range of applications, including, chemical concentration sensing [9], liquid level sensing [10] and as a means of forming a tuneable spectral filter [11]. A TFBG produces numerous resonances in the transmission spectrum of the optical fibre, the monitoring of which has been used to demonstrate a refractometer [12]. A Fresnel based refractometer is used to evaluate the sensors and the results are briefly compared with two traditional cure monitoring methods, differential scanning calorimetry (DSC) and impedance spectroscopy.

* r.p.tatam@cranfield.ac.uk; phone: +44 (0)1234 754630; fax: +44 (0)1234 750728; www.cranfield.ac.uk/soe/cpoe 


\subsection{Long Period Gratings}

A long period grating (LPG) consists of a periodic refractive index modulation of the core of an optical fibre. The period of the modulation is typically in the range $100 \mu \mathrm{m}$ to $1000 \mu \mathrm{m}$, and supports coupling between co-propagating modes of the optical fibre. In the case of single mode fibre, the coupling takes place between the guided mode and co-propagating cladding modes. Efficient coupling is possible to only a subset of these cladding modes [8]. As the cladding is a poor waveguide, the modes are lossy and the transmission spectrum of an optical fibre containing an LPG contains a number of attenuation bands, each corresponding to coupling to a different cladding mode. The phase matching wavelengths are governed by the expression [13],

$$
\lambda_{i}=\left\lfloor n_{\text {eff }} c o(\lambda)-n_{\text {eff_cl }}^{i}(\lambda)\right\rfloor \Lambda
$$

where $\lambda_{\mathrm{i}}, \mathrm{n}_{\text {eff_co }}, \mathrm{n}_{\text {eff_cl }}^{\mathrm{i}}$ and $\Lambda$ are the $\mathrm{i}^{\text {th }}$ cladding mode resonance wavelength, effective index of the core, effective index of the $\mathrm{i}^{\text {th }}$ cladding mode, and the grating period respectively. The refractive index sensitivity of LPGs arises from the dependence of the coupling wavelength upon the effective index of the cladding mode. The response of an LPG to refractive index is evident as a shift in the central wavelength of the attenuation bands; this is shown in figure 1. The higher order modes show an increased sensitivity, and this is enhanced as the refractive index approaches that of the cladding. When the refractive index matches that of the cladding, the cladding modes are no longer supported as the cladding appears to be of infinite thickness. Further increase of the refractive index leads to the bands reappearing, corresponding to the existence of leaky modes, but at a longer wavelength and with significantly reduced refractive index sensitivity [13].

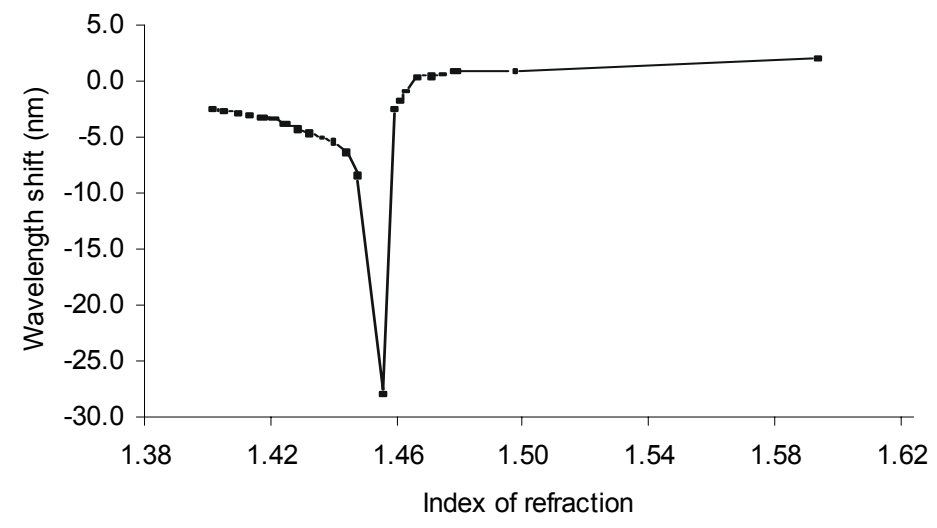

Figure 1. Plot of the wavelength shift of an attenuation band against the index of refraction of the material surrounding an LPG of period $400 \mu \mathrm{m}$, written in boron-germanium co-doped fibre with a cut-off wavelength of $650 \mathrm{~nm}[8]$.

The LPG response to changes in the surrounding refractive index, shown in figure 1, was characterised by immersing the section of fibre containing the LPG in a series of Cargille refractive index oils of differing refractive index. The refractive index at the wavelength of operation was calculated using the Sellmeier equation. The wavelength response was then fitted with a 6 th order polynomial to provide a function relating wavelength shift to refractive index.

\subsection{Tilted Fibre Bragg Gratings}

A standard fibre Bragg grating (FBG) consists of a refractive index modulation in the core of an optical fibre that acts to couple the fundamental forward propagating core mode to the contra-propagating core mode. A tilted FBG (TFBG) [14, 15] consists of a refractive index modulation that is purposely tilted or blazed relative to the fibre axis in order to enhance coupling between the forward-propagating core mode and contra-propagating cladding modes, as illustrated in 
figure 2. The contra-propagating cladding modes are poorly guided and attenuate rapidly and are therefore not noticeable in reflection but are observed as numerous resonant bands in the transmission spectrum of the TFBG. A typical transmission spectrum is shown in figure 3.

The spectral response of the TFBG is governed by the phase matching condition:

$$
\lambda_{i}=\left(n_{\text {eff_co }}+n_{\text {eff_cl }}^{i}\right) \cdot \Lambda_{\mathrm{B}}, \quad \text { where } \Lambda_{\mathrm{B}}=\frac{\Lambda}{\cos \theta}
$$

where $\lambda_{\mathrm{i}}, \mathrm{n}_{\text {eff co }}, \mathrm{n}_{\text {eff_cl }}^{\mathrm{i}}, \theta, \Lambda$ and $\Lambda_{\mathrm{B}}$ are the $\mathrm{i}^{\text {th }}$ cladding mode resonance wavelength, effective index of the core, effective index of the $i^{\text {th }}$ cladding mode, tilt angle, grating period and blaze period respectively.

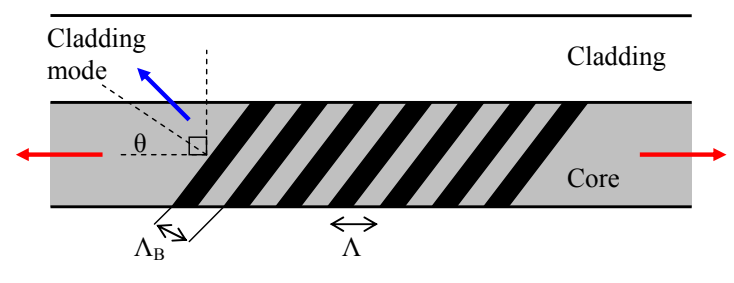

Figure 2. Schematic diagram of a tilted fibre Bragg grating (TFBG). $\Lambda$; grating period, $\Lambda_{\mathrm{B}}$; blaze period and $\theta$; blaze angle.

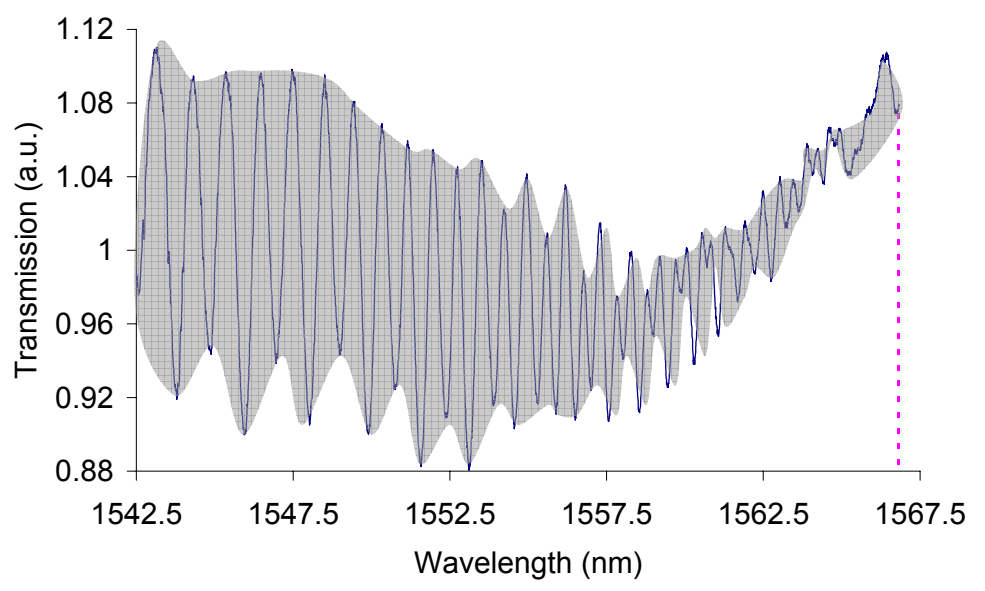

Figure 3. Transmission spectrum of a TFBG of length $5 \mathrm{~mm}$ and blaze angle $4.5^{\circ}$, fabricated in boron-germanium codoped optical fibre (Fibercore PS1250). The dotted line indicates the position of the Bragg wavelength. The shaded area illustrates the envelope technique used for area calculation.

The TFBG spectral resonances are more complex and require a different method of analysis compared to that of the LPG. One method by which this may be achieved is by constructing an envelope that completely surrounds the spectral resonances [12]. The area that is enclosed by the envelope varies with changes in the refractive index of the surrounding environment. By monitoring this change and relating it to the refractive index it is possible to use the TFBG as a refractometer. The TFBG response to changes in surrounding refractive index, shown in figure 4, was characterised by immersing the section of fibre containing the TFBG in a series of Cargille refractive index oils of differing refractive index. The refractive index at the wavelength of operation was calculated using the Sellmeier equation. The wavelength response was then fitted with a 3rd order polynomial, relating wavelength shift to refractive index. 


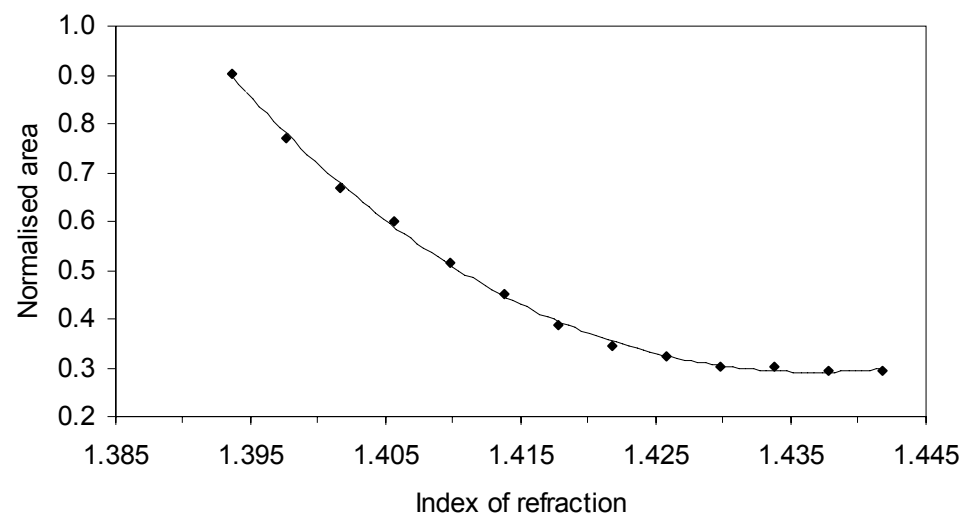

Figure 4. Normalised area of the envelope of the TFBG resonances (with reference to that when the TFBG is in air) plotted as a function of the refractive index of the surrounding medium.

In this paper, the use of LPGs and TFBGs as resin cure monitoring sensors is investigated. The index of refraction of a polymerising resin changes as the molecular density increases and as the atomic bonds involved in the polymerisation processes are altered [16]. The sensitivities of TFBGs and LPGs to changes in refractive index are exploited with the aim of developing a method of measuring the degree of cure. To aid cross comparison of results, a Fresnel reflection based refractometer has been designed and implemented [17]. The resin cure is characterised further using traditional techniques of cure monitoring; differential scanning calorimetry (DSC) and impedance spectroscopy. These are compared with the grating methods described above. As a further extension of the LPG technique, preliminary results are also shown utilising the LPG sensor as a Fresnel based refractometer. This may indicate the possible use of LPGs with resin systems of refractive indices greater than that of the sensing fibre.

\section{EXPERIMENT}

A TFBG of length $5 \mathrm{~mm}$ and blaze angle $4.5^{\circ}$ was fabricated in boron-germanium co-doped optical fibre, Fibercore PS1250 with a cut-off wavelength of $1240 \mathrm{~nm}$, using the near-field interference pattern of a tilted phase-mask. The photosensitivity of the fibre was enhanced by pressurizing it in hydrogen for a period of 2 weeks at a pressure of $150 \mathrm{bar}$ at room temperature. The phase mask was illuminated with a UV beam at a wavelength of $248 \mathrm{~nm}$ and with an average power of $40 \mathrm{~mW}$. The TFBG was interrogated by coupling the output from a super-luminescent diode of bandwidth $60 \mathrm{~nm}$ into the optical fibre, and monitoring the transmission using a scanning fibre Fabry-Perot interferometer of free spectral range $43 \mathrm{~nm}$ and of finesse 900 [18].

To investigate the LPG's response to the refractive index change of the resin at different wavelengths, LPGs of length $40 \mathrm{~mm}$ and $400 \mu \mathrm{m}$ period were fabricated in two different fibre types, one with a cut off wavelength of $650 \mathrm{~nm}$ (Fibercore SM750) and the other with a cut off of 1275nm (Optical Fibres 1310). The fibre was photosensitised by pressurising it in hydrogen for a period of 2 weeks at a pressure of $150 \mathrm{bar}$ at room temperature. The fibre was then placed behind an amplitude mask that was illuminated by a UV laser beam at a wavelength of $266 \mathrm{~nm}$, provided by a frequency-quadrupled $\mathrm{Nd}$ :YAG laser.

The transmission spectrum of the fibre with the shorter cut-off wavelength, shown in figure 5, was monitored by coupling the output from a tungsten-halogen white light source into the fibre and coupling the transmitted light to a CCD spectrometer (Ocean Optics S2000) with a resolution of $0.3 \mathrm{~nm}$. The transmission spectrum of the fibre with the longer cut-off wavelength was monitored using an Advantest Q8381 spectrum analyser with a resolution of $0.1 \mathrm{~nm}$. 


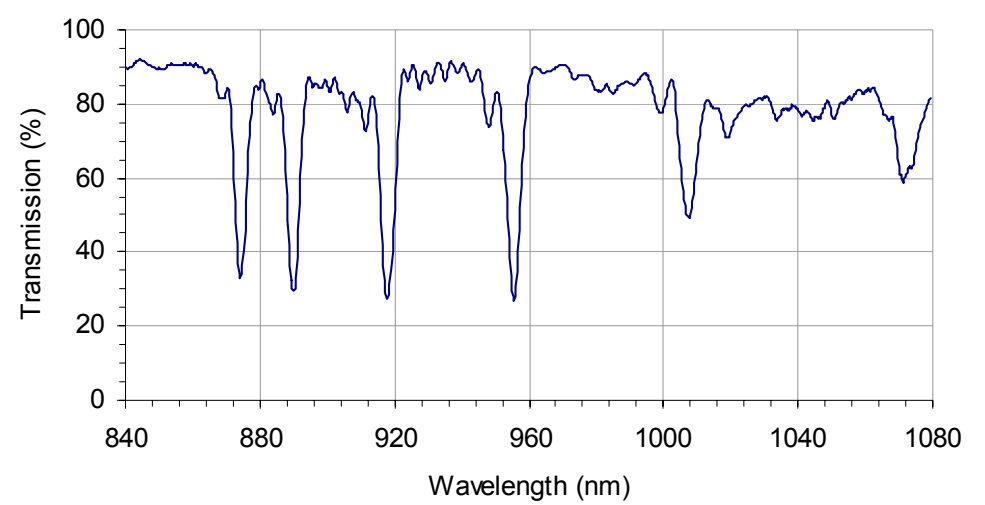

Figure 5. Transmission spectrum of an LPG of length $40 \mathrm{~mm}$ and of period of $400 \mu \mathrm{m}$, fabricated in an optical fibre with a cut-off wavelength of $650 \mathrm{~nm}$ (Fibercore SM750).

The responses of the fibre grating based refractometers to the refractive index change were compared with refractive index measurements made using a Fresnel based refractometer [17]. Two Fresnel refractometers were constructed, operating at wavelengths of $833 \mathrm{~nm}$ and $1575 \mathrm{~nm}$. This was to aid correlation with the wavelength regions used by the fibre gratings and to reduce the differences introduced by the dispersion of the resin. The Fresnel approach is complementary to the grating sensors in offering a highly localised measurement of the refractive index change at the end of a fibre that can be readily calibrated, as described below. However, this approach is not as appropriate for applications where multiplexing of several sensors is required, or where a longer gauge length sensor is necessary.

\subsection{Fresnel Refractometer}

The experimental configuration is shown in figure 6. The output from a laser diode operating at the appropriate wavelength was intensity modulated at a frequency of $270 \mathrm{~Hz}$, and coupled into a network of $3 \mathrm{~dB}$ directional couplers. The Fresnel reflection from the fibre/resin interface was monitored using photodiode $\mathrm{pd}_{1}$. In the second arm, photodiode $\mathrm{pd}_{2}$ monitored the reflection from the air/fibre interface, acting as an intensity reference to aid the normalisation of the signal and to account for any change in power coupled into the optical fibre from the laser diode. The outputs from the photodiodes were monitored using lock-in amplifiers. To minimize contributions from unwanted Fresnel reflections from the unused port of the $3 \mathrm{~dB}$ coupler, micro bends were induced in the fibre to form a high attenuation return path for the reflections.

The refractive index determined using the refractometer is given by [17]

$$
\begin{gathered}
n=n_{\text {eff_co }} \times \frac{1-\frac{\Delta}{\sqrt{R}}}{1+\frac{\Delta}{\sqrt{R}}} \\
\Delta=\frac{n_{\text {eff_co }}{ }^{-n_{a}}}{n_{\text {eff _c }}{ }^{+n_{a}}} \\
R=\frac{V_{\text {air }}}{V_{\text {epoxy }}}
\end{gathered}
$$

where, $n_{\text {eff co }}$ is the effective refractive index of the fibre mode, na is the refractive index of air given as 1.0002739 [17], $\mathrm{V}_{\text {air }}$ is the voltage produced by photodiode $\mathrm{pd}_{2}$, obtained for the reflection from the fibre/air interface and $\mathrm{V}_{\text {epoxy }}$ is the voltage produced by photodiode $\mathrm{pd}_{1}$, corresponding to the reflection from the fibre/resin interface. 
A UV cured epoxy resin, EpoTek OG134, with an uncured refractive index of 1.4163, measured at 589nm [19], was used as the test resin. A UV lamp, with output power $4 \mathrm{~W}$ and with a spectral peak at $365 \mathrm{~nm}$, was positioned to provide a fluence of $6.57 \mathrm{~mW} / \mathrm{cm}^{2}$ in order to cure the resin slowly and to allow the reaction to be recorded using the relatively low sampling rate achievable using the OSA. The LPG and TFBG spectra and the reflection signal from the Fresnel probe were recorded at $0.5 \mathrm{~s}$ intervals during the cure.

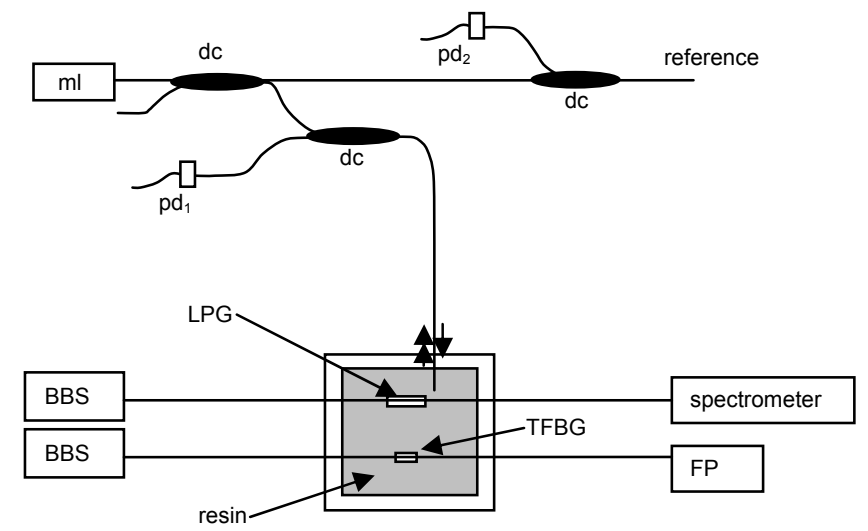

Figure 6. Experimental configuration. ml, modulated laser diode light source; pd, photodiode; dc, directional coupler; BBS, broadband source; FP, scanning Fabry-Perot interferometer; LPG, long period grating; TFBG, tilted fibre Bragg Grating.

\subsection{Differential scanning calorimetry and impedance spectroscopy}

To assess how indicative the refractive index change during cure was to the resins cure state it was necessary to compare the results with standard assessment techniques. Two methods were investigated, differential scanning calorimetry (DSC) and impedance spectroscopy.

DSC is a widely used technique in the materials industry for investigating the characteristics of polymers. It is a method that measures the heat flow absorbed or emitted by the sample. In the case of thermosetting curing, the heat flow is proportional to the rate of reaction [20]. Normalisation and integration of the heat flow versus time curve using an appropriate baseline [21] yields the degree-of-cure evolution with time. A sample was prepared using the PTFE well and UV cured at a constant temperature of $29^{\circ} \mathrm{C}$ in a TA Instruments $2920 \mathrm{MDSC}$ with an RCS cooling unit. The resin was irradiated under the same conditions as the refractometry experiments.

Impedance spectroscopy is proposed as an online method for monitoring a polymer during cure [22, 23]. It is based on changes in the electrical and dielectric properties as a polymer cure progresses. The molecular structure of a material undergoes significant change during cure and the material goes through phase transitions in the cure cycle, i.e. liquid to rubber to glass. Much information can be derived from impedance measurements but for the purposes of on line monitoring in a control context it is necessary to relate a dielectric parameter directly to a material state index. In this experiment the imaginary impedance maximum (IIM) is used as a dielectric parameter to asses the cure state of the epoxy under cure. A sample was prepared and interrogated using a Solartron Instruments 1260 frequency response analyzer over a frequency range, with a GIA (Pearson Panke) interdigitated dielectric sensor. The analysis proposed in [18] was followed to assess the cure progression from the impedance data. As for the refractometry experiments, the resin was irradiated under the same conditions.

\section{RESULTS}

Figure 7 shows the refractive index of the curing resin measured by both of the Fresnel based refractometers (solid line $833 \mathrm{~nm}$ and dashed line $-1575 \mathrm{~nm}$ ). The datum is the time at which the UV lamp was switched on. At this time a clear decrease in refractive index was observed. This is due to an exothermic reaction initiated by the incident UV light. The temperature of the resin was measured with a thermocouple, placed in the resin, which indicated a $6.5^{\circ} \mathrm{C}$ increase in 
temperature over the first 5 minutes. It was also noted that there was an ambient temperature increase of $3^{\circ} \mathrm{C}$. This was measured with a thermocouple placed close to the sample. The thermocouple measurements are shown in figure 8 .

The measurements indicating the evolution of the refractive index made at the two wavelengths show good agreement. After the initial decrease in the refractive index, a rapid increase is measured, indicating the reaction process. The rate of change of refractive index reduces after 90 minutes. The difference between the measurements made at the two wavelengths may be due to the dispersion of the resin.

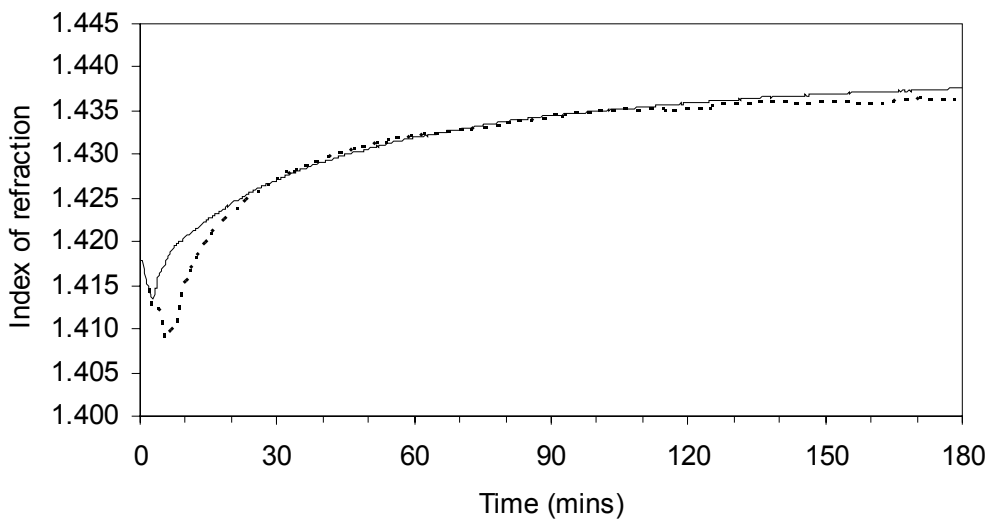

Figure 7. Refractive index change measured during the cure of the OG-134 epoxy resin, determined from the measurements made using the Fresnel refractometers operating at $833 \mathrm{~nm}$ (solid) and $1575 \mathrm{~nm}$ (dashed)

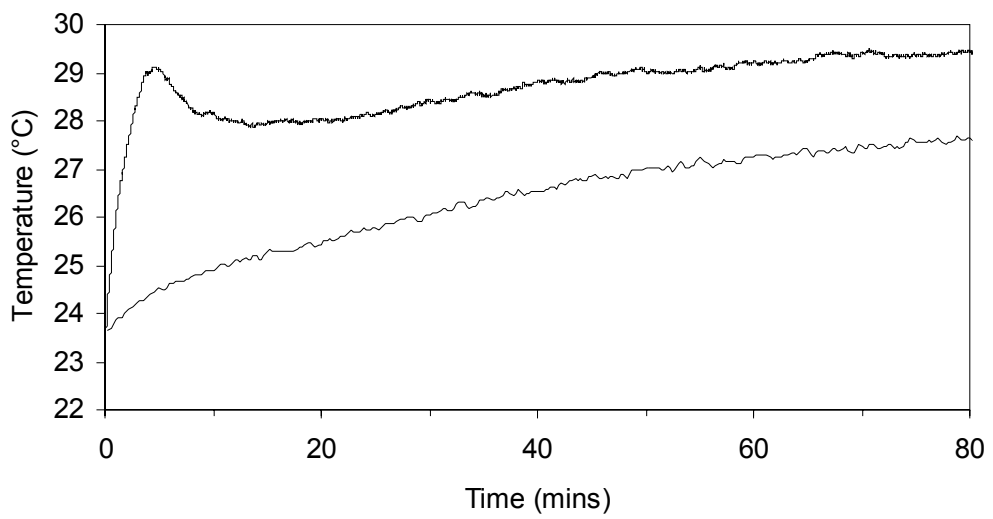

Figure 8. Temperature of the OG-134 epoxy resin measured during cure by two K-type thermocouples; one placed in the sample (solid) and the second placed close to the sample (dashed). An exothermic peak is evident shortly after the UV lamp was turned on.

A simple model was used to predict the expected wavelength shift of an LPG band in response the refractive change during cure. The model uses Gloge's weakly guiding approximation [24] to calculate the effective refractive index of the core mode. The standard dispersion equation for a step index fibre [25] was used to calculate the effective indices of the cladding modes. The central wavelengths of the attenuation bands may then be determined using equation (1). The refractive index measured during the cure of the resin using the Fresnel system was then input to the model to calculate the corresponding change in the cladding mode effective index, and thus allow prediction of the change in the LPG attenuation band wavelength. Figure 9 shows the predicted shift of the central wavelength of the longest wavelength $\left(5^{\text {th }}\right)$ attenuation band of an LPG fabricated in the lower cut-off wavelength fibre, of length 40mm and with a $400 \mu \mathrm{m}$ period. Shown here also is the experimentally measured response of an LPG of the same design. The close agreement with the 
model shows the potential for using an LPG as a cure monitoring sensor. The contributions of strain and temperature to the measured wavelength shift are not included in the simplified model. Transverse strain is known to develop during the cure for some resin systems [18], but has not yet been determined for the UV resin used here.

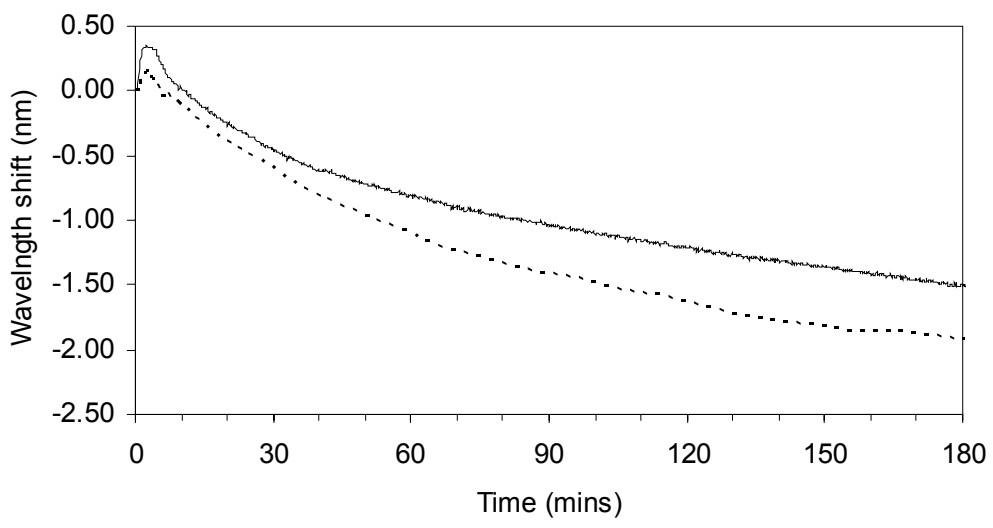

Figure 9. The experimental (solid) and theoretical (dashed) wavelength response of the $5^{\text {th }}$ attenuation band of an LPG (central wavelength at $940 \mathrm{~nm}$ ), of length $40 \mathrm{~mm}$ and of period $400 \mu \mathrm{m}$ fabricated in a low cut-off wavelength fibre (SM750), during the cure of the OG-134 epoxy resin.

Using the calibration method discussed in section 1.1, the wavelength responses of the LPGs were related to refractive index. Figure 10 shows the refractive index calculated using the fifth attenuation bands of both LPGs (SM750 and OF1310 fibre). At both wavelengths the initial decrease in refractive index, observed when the UV lamp is switched on, is followed by a rapid positive change in refractive index. The LPGs show a decrease in the rate of change of refractive index after 90 minutes, in agreement with the measurements made using the Fresnel refractometer. The discrepancies may be attributed to dispersion effects and to errors induced in the calibration, where the thermo-optic change in refractive index of the resin was assumed to be negligible. The results obtained using both the Fresnel technique and the LPG based method indicates that the onset of the positive refractive index change is delayed when the measurement is made at the longer wavelength. This may point to the dispersive properties of the resin. The refractive index of the resin at the shorter wavelengths $(\sim 800 \mathrm{~nm})$ approaches the high sensitivity regions of the refractometers before that of the longer wavelengths $(\sim 1550 \mathrm{~nm})$.

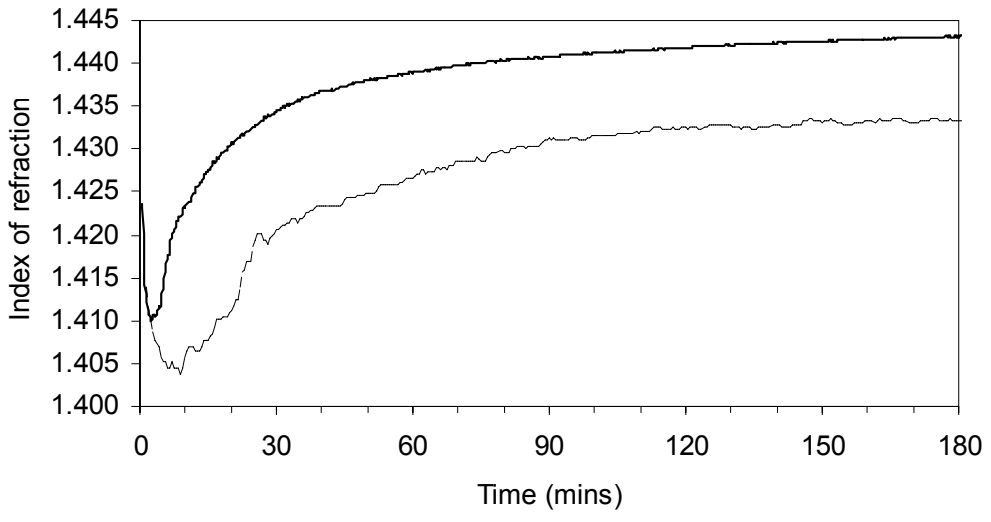

Figure 10. The evolution of the refractive index during cure of the OG-134 epoxy resin, determined from the wavelength shift of the $5^{\text {th }}$ attenuation bands of the LPG based refractometers at $940 \mathrm{~nm}$ (solid) and $1545 \mathrm{~nm}$ (dashed). 
Tables 1 and 2 compare the measurements from the LPG and Fresnel techniques in the two wavelength regimes.

Table 1. Comparison of the refractive index change exhibited by EpoTek OG134 during cure, determined from the Fresnel $(833 \mathrm{~nm})$ and LPG (fabricated in the PS750 fibre) methods. ( $\Delta$ is the difference between the measurements made using the two methods).

\begin{tabular}{l|l|l|l}
\cline { 2 - 4 } \multicolumn{2}{c}{ Optical technique } \\
\hline Cure state & Fresnel & LPG & $\Delta$ \\
\hline Wet (initial) & 1.4178 & 1.4237 & 0.0059 \\
Cured (final) & 1.4376 & 1.4432 & 0.0056 \\
\hline
\end{tabular}

Table 2. Comparison of the refractive index change exhibited by EpoTek OG134 during cure, determined from the Fresnel $(1575 \mathrm{~nm})$ and LPG (fabricated in the OF1310 fibre) methods. ( $\Delta$ is the difference between the measurements made using the two methods).

\begin{tabular}{l|l|l|l}
\cline { 2 - 4 } \multicolumn{2}{c}{ Optical technique } \\
\hline Cure state & Fresnel & LPG & $\Delta$ \\
\hline Wet (initial) & 1.4184 & 1.4190 & 0.0006 \\
Cured (final) & 1.4365 & 1.4332 & -0.0033 \\
\hline
\end{tabular}

The refractive index change determined over the cure cycle by the TFBG is shown in figure 11 . The form of the curve agrees well with the results from the Fresnel and LPG based refractometers. The refractive index starts to increase 5 minutes after the UV lamp is switch on. The TFBG sensor indicates a refractive index of 1.437 when the resin is fully cured, achieved after 180 minutes. This agrees, to within $5 \times 10^{-4}$ rius (refractive index units), with the measurement from the Fresnel refractometer operating at $1575 \mathrm{~nm}$. The calibration discussed in section 1.2 revealed that the sensitivity of the TFBG decreases rapidly for refractive indices greater than 1.43, and it is apparent that the response curve starts to flatten after 60 minutes as the TFBG starts to lose sensitivity. All three sensor types show that most of the reaction takes place in the first 90 minutes, after which there is a gradual reduction in the rate of change of refractive index. The reaction is almost complete after 2 hours.

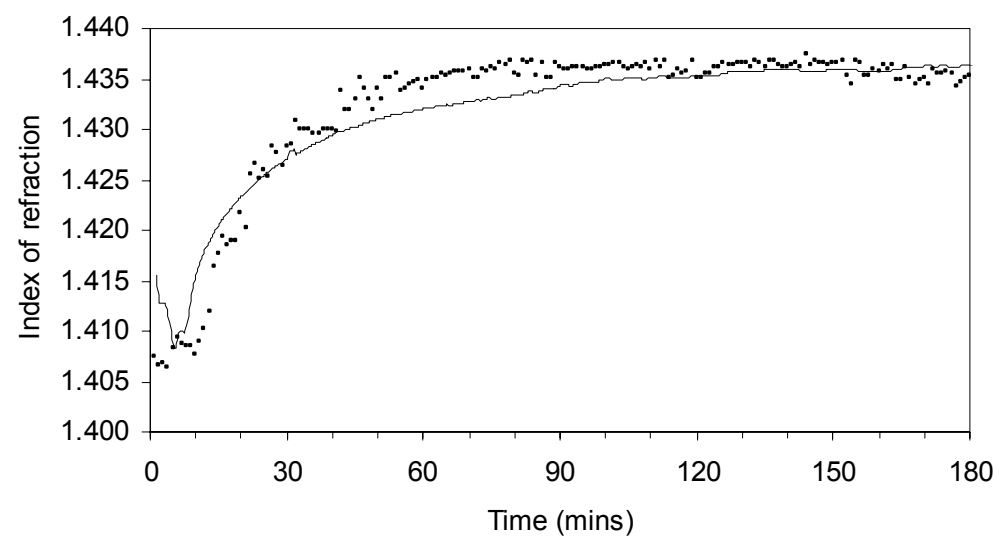

Figure 11. Tilted Fibre Bragg Grating (TFBG) calibrated response to refractive index change at 1550nm for the OG-134 UV cured epoxy during cure(-) The Fresnel response at $1575 \mathrm{~nm}$ (dashed) is also shown for comparison. 
The performances of the different techniques are summarised in table 3 . The discrepancy between results obtained using the Fresnel and grating based refractometers may be attributed to the temperature and strain sensitivities of the LPG and TFBG sensors, as mentioned previously. The thermo-optic effect may also have an influence on the resonant wavelength, equations (1) and (2). This effect is negligible for the Fresnel technique. Investigation into the influence of these effects is the subject of current work.

Table 3. Comparison of refractometer methods (depending on fibre type).

\begin{tabular}{lll}
\hline Refractometer & Gauge Length & Sensitivity Range \\
\hline LPG & $10-1000 \mathrm{~mm}$ & $1.400-1.456$ \\
TFBG & $0.1-12 \mathrm{~mm}$ & $1.405-1.437$ \\
Fresnel & $2-10 \mu \mathrm{m}$ & Air- 1.456 \\
\hline
\end{tabular}

A direct comparison of the calculated refractive index change during cure and the results from both the DSC and impedance spectroscopy are shown in figures 12 and 13. Both results show very good agreement. It illustrates how a grating based refractometer may be used to provide data for process monitoring of complex composite components. DSC or spectroscopic based kinetic modelling can be correlated with on-line grating data to determine a state of cure.

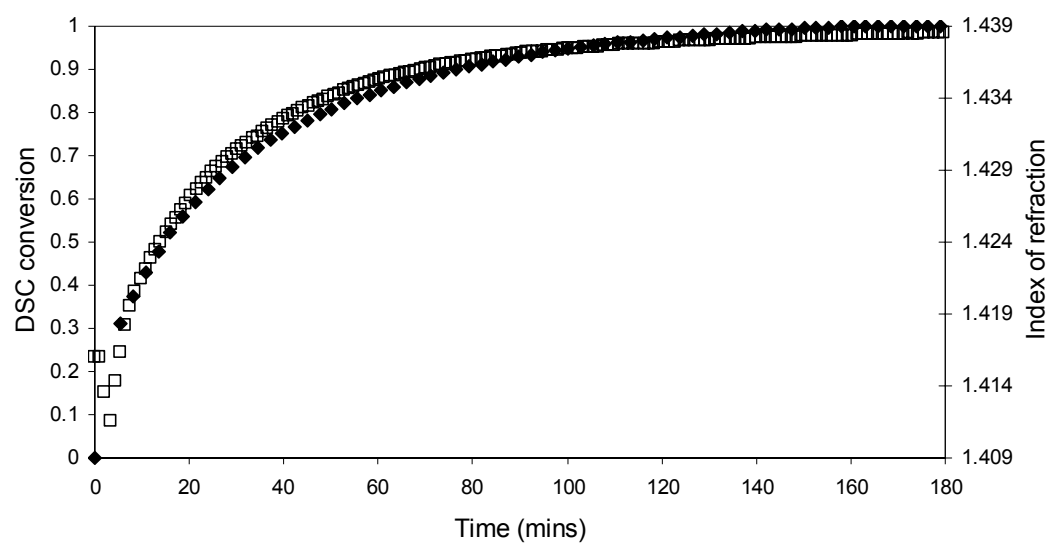

Figure 12. Index of refraction $(\square)$ and DSC conversion $(\bullet)$ of UV cured epoxy resin. 


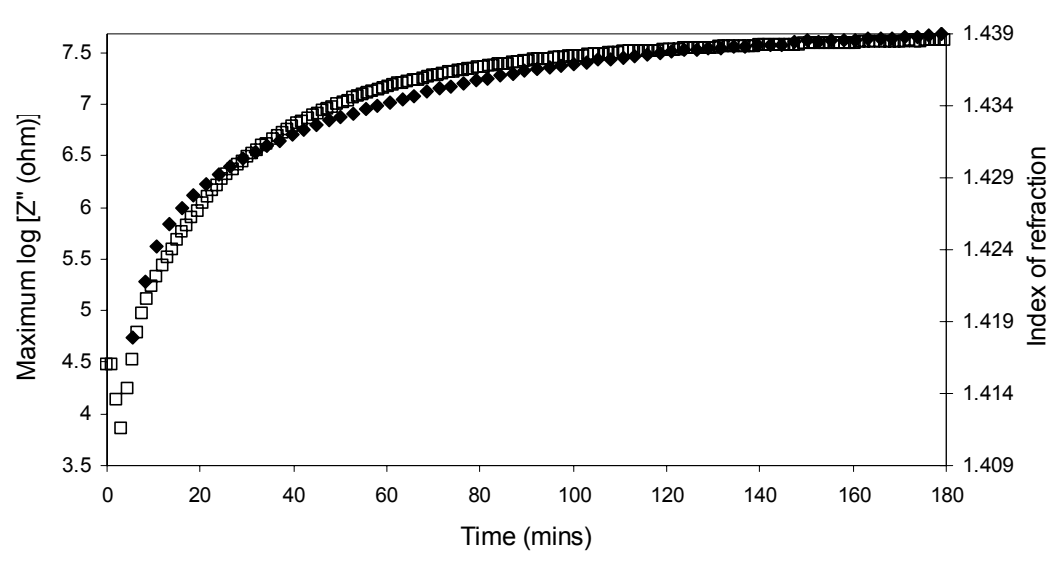

Figure 13. Index of refraction $(\square)$ and impedance spectroscopy measurements $(\bullet)$ of UV cured epoxy resin.

\subsection{High index LPG sensor}

The wavelength response of the LPG attenuation bands to refractive index (figure 1) exhibits a region with high sensitivity, which corresponds to refractive indices close to that of the cladding of the sensing fibre. At indices higher than that of the cladding, the wavelength response is shown to be significantly reduced. This places a requirement on the fibre design; that the fibre index must closely match the index of the resin system. Most structural resin systems however have a refractive index that is higher than that of standard silica optical fibre. Here, to extend the measurement range to refractive indices higher than that of the cladding, we propose to exploit the refractive index dependence of the extinction ratio of the LPG attenuation bands to the external refractive index.

For surrounding indices higher than that of the cladding, propagating cladding modes are no longer supported. However, leaky modes associated with Fresnel reflection at the glass/surround interface are evident as attenuation bands with a slightly longer wavelength and a reduced extinction ratio. These attenuation bands show a very weak wavelength sensitivity to changes in the surrounding index greater than that of the cladding but the extinction ratios have been shown to increase as the surrounding refractive index increases beyond that of the cladding [13]. The preliminary results for an isothermal cure of an epoxy with an initial uncured refractive index of 1.53 are shown in figure 13. The resin was isothermally cured at $180^{\circ} \mathrm{C}$ over two hours. The refractive index change during cure was calculated using the Fresnel refractometry method, discussed in section 2.1. The change in extinction ratio of the LPG attenuation band shows good correlation with the measured change in refractive index. The Fresnel signal is noisy during the early stages of the cure, which may be a result of volatiles degassing from the sample. The results illustrate the effectiveness of the use of an LPG as a cure sensor for resins with a high refractive index. The use of LPGs in standard silica fibre may also be employed as resin location sensors in a composite component, where the change in the extinction ratio of an attenuation band may be indicative of the presence of resin. 


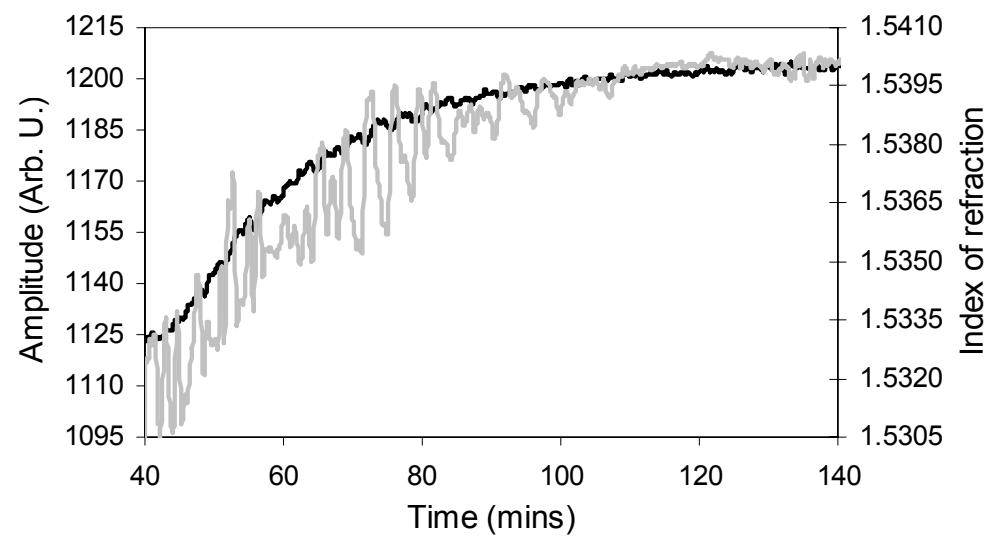

Figure 13. Extinction ratio (-) of the $5^{\text {th }}$ attenuation band of a $650 \mathrm{~nm}$ cut-off fibre, $400 \mathrm{~mm}$ period LPG of $40 \mathrm{~mm}$ length and refractive index change $(-)$ calculated with a Fresnel refractometer at $1575 \mathrm{~nm}$

\section{SUMMARY}

In summary, optical fibre refractometers have been employed to monitor the refractive index changes occurring in a curing epoxy resin. The core-cladding mode coupling resonances of a tilted fibre Bragg grating (TFBG) and of an LPG have been shown to be sensitive to the cure of an epoxy resin. The measurements made using the grating refractometers were compared with those made using a fibre optic Fresnel reflection based technique, and were found to be in agreement to within $6 \times 10-4$ rius. The grating based refractometers are sensitive to temperature and strain, which must be decoupled to accurately measure refractive index. The results are also compared with traditional cure monitoring techniques and show close agreement. This leads to the possibility of using the sensors with cure-kinetic models to characterise and monitor the cure of resins and fibre reinforced resin systems [26]. The change in extinction ratio of LPG bands to high index resins may also provide a method for monitoring the cure of resins.

The authors gratefully acknowledge the support for this work from the Engineering and Physical Sciences Research Council (EPSRC), UK.

\section{REFERENCES}

1. B. Degamber and G. F. Fernando, "Process monitoring of fiber-reinforced polymer composites," MRS Bulletin 27, 370-380 (2002).

2. J. Mijovic and S. Andjelic, "In situ real-time monitoring of reactive systems by remote fibre-optic near-infra-red spectroscopy," Polymer 36, 3783-3786 (1995).

3. J. R. Dunkers, K. M. Flynn, M. T. Huang and W. McDonough, "Fourier transform near-infrared monitoring of reacting resins using an evanescent wave high-index fiber-optic sensor" Applied Spectroscopy 52, 552-556 (1998).

4. R. L. Levy and S. D. Schwab, "Monitoring the composite curing process with a fluorescence-based fiber-optic sensor," Polymer Composites 12, 96-101 (1991).

5. D. L. Woederman, K. M. Flynn, J. P. Dunkers and R. S. Parnas, "The use of evanescent wave fluorescence spectroscopy for control of the liquid molding process," J. Reinforced Plastics and Composites 15, 922-943 (1996).

6. D. L. Woederman, J. K. Spoerre, K. M. Flynn and R. S. Parnas, "Cure monitoring of the liquid composite molding process using fiber optic sensors," Polymer Composites 18, 133-150 (1997)

7. T. Takeo and H. Hattori, "Optical Fiber Sensor for Measuring Refractive Index," Japan J. Appl. Phys. 21, 15091512 (1982).

8. S. W. James and R. P. Tatam "Optical Fibre Long Period Grating Sensors: Characteristics and Application," Measurement Science and Technology 14, 49-61 (2003). 
9. T. Allsop, L. Zhang and I. Bennion, "Detection of organic aromatic compounds in paraffin by a long-period fiber grating optical sensor with optimized sensitivity," Optics Communications 191, 181-190 (2001).

10. S. Khaliq, S. W. James and R. P. Tatam, "Fibre optic liquid level sensor using a long period grating," Optics Letters 6, 1224-1226 (2001).

11. Min-Suk Kwon and Sang-Yung Shin, "Tunable notch filter using a thermo-optic long-period grating," Journal of Lightwave Technology 22, 1968-1975 (2004).

12. G. Laffont and P. Ferdinand, "Tilted short-period fibre-Bragg-grating-induced coupling to cladding modes for accurate refractometry," Measurement Science and Technology 12, 765-770 (2001).

13. H. J. Patrick, A. D. Kersey F. and Bucholtz "Analysis of the Response of Long Period Fiber Gratings to External Index of Refraction," Journal of Lightwave Technology 16, 1606-1612 (1998).

14. T. Erdogan and J. E. Sipe, "Tilted fiber phase gratings," J. Optical Society of America A 13, 296-313 (1996).

15. L. Brilland, D. Pureur, J. F. Bayon and E. Delevaque E, "Slanted gratings UV-written in photosensitive cladding fibre," Electronics Letters 35, 234-236 (1999).

16. Z. Boming, W. Dianfu Du. Shanyi and S. Yinlin, "An investigation of a fiber optic sensor in the composite cure process," Smart Materials and Structures 8, 515-518 (1999).

17. C. B. Kim and C. B. Su, "Measurement of the refractive index of liquids at 1.3 and 1.5 micron using a fibre optic Fresnel ratio meter," Measurement Science and Technology 15, 1683-1686 (2004).

18. C-C. Ye, S. E. Staines, S. James and R. P. Tatam, "A polarisation maintaining fibre Bragg grating interrogation system for multi-axis strain sensing," Measurement Science and Technology 13, 1446-1449 (2002).

19. Epoxy Technology, http://www.epotek.com

20. R. B. Prime, "Differential Scanning Calorimetry of the epoxy cure reaction," Poly. Eng. Sci. 13, (5) 365-371 (1973).

21. U. Bandara, "A systematic solution to the problem of sample background correction in DSC curves," Journal of Thermal Analysis 31, 1063 (1986).

22. A. Maffezzoli, A. Trivisano, M. Opalicki, J. Mijovic and J. M. Kenny "Correlation between dielectric and chemorheological properties during cure of epoxy-based composites," J. of Mat. Science 29, 800-808 (1994).

23. A. A. Skordos and I. K. Partridge, "Determination of the degree of cure under dynamic and isothermal curing conditions with electrical impedance spectroscopy," J. of Poly. Sci: B: Polymer Physics 42, 146-154 (2004).

24. D. Gloge, "Weakly guiding fibers," Applied Optics 10, 2252-2258 (1971).

25. A. Yariv, Optical electronics in Modern Communications, Oxford University Press, 1996.

26. E. Chehura, A. A. Skordos, S. W. James, I. K. Partridge and R. P. Tatam "Strain development in curing epoxy resin and glass fibre/epoxy composites monitored by fibre Bragg grating sensors in birefringent optical fibre," Smart Materials and Structures 14, 354-362 (2005). 